\title{
Investigation of the Lattice Boltzmann SRT and MRT Stability for Lid Driven Cavity Flow
}

\author{
E. Aslan, I. Taymaz, and A. C. Benim
}

\begin{abstract}
The Lattice Boltzmann Method (LBM) is applied to incompressible, steady, laminar flow high Reynolds numbers varying in a range from 200 to 2000 for determining stability limits of the LBM Single Relaxation Time (LBM-SRT) and the LBM Multiple Relaxation Time (LBM-MRT). The lid driven cavity flow is analyzed. The effect of the model Mach number on accuracy is investigated by performing computations at different Mach numbers in the range $0.09-0.54$ and comparing the results with the finite-volume predictions of the incompressible Navier-Stokes equations. It is observed that the Mach number does not affect the results too much within this range, and the results agree well with the finite volume solution of the incompressible Navier-Stokes equation. LBM-MRT is more stable than LBM-SRT especially for low Mach and high Reynolds numbers. For the LBM-SRT solutions, collision frequency $(\omega)$ decreases with increasing Reynolds and Mach numbers, however, for the LBM-MRT solutions, 7th and 8th relaxation rates $\left(s_{7}=s_{8}\right)$ decrease with decreasing Reynolds numbers and with increasing Mach numbers. Within its stability range, the convergence speed of the LBM-SRT is higher (approximately \%10) than that of LBM-MRT, while the convergence speed of the finite volume method is much lower than the both LBM formulations (the LBM-SRT and the LBM-MRT).
\end{abstract}

Index Terms-Lid driven cavity flow, lattice Boltzmann method, single relaxation time, multiple relaxation time.

\section{INTRODUCTION}

The Lattice Boltzmann equation (LBE) using relaxation technique was introduced by Higuera and Jimenez [1] to cope some drawbacks of Lattice Gas Automata (LGA) such as large statistical noise, limited range of physical parameters, non-Galilean invariance and difficult implementation in three dimension problem [2]. In the original derivation of LBE using relaxation concept, it was strongly connected to the underlying LGA. However, it was soon recognized that it could be constructed independently [3]. After that, the Lattice Boltzmann Method (LBM) has received considerable attention as an alternative to conventional computational fluid

Manuscript received February 5, 2014; revised April 4, 2014.

E. Aslan is with the Department of the Mechanical Engineering, Istanbul University, 34320, Istanbul, Turkey (e-mail: erman.aslan@istanbul.edu.tr).

I. Taymaz is with the Department of the Mechanical Engineering, Sakarya University, 54187, Sakarya, Turkey (e-mail: taymaz@sakarya.edu.tr).

A.C. Benim is with the CFD Lab., Department of Mechanical and Process Engineering, Duesseldorf University of Applied Sciences, Josef-Gocklen Str.9, 40474, Duesseldorf, Germany (e-mail: alicemal.benim@fh-duesseldorf.de). dynamics for simulation complex flow problems.

The simplest LBE is the Lattice Bhatnagar-Groos-Krook (LBGK) equation, based on a Single Relaxation Time (LBM-SRT) approximation [4]. Due to extreme simplicity, the LBGK equation has become most popular Lattice Boltzmann equation in spite of its well-known deficiencies, for example, flow simulation at high Reynolds numbers [5].

Flow simulation at high Reynolds numbers, collision frequency $(\omega)$ which is the main ingredient of the LBM-SRT, exhibits a theoretical upper bound $(\omega<2)$ that is related with the positiveness of the molecular kinematic viscosity [6]. Thus, stability problems arise as the collision frequency approaches to this limiting value [7]. For incompressible flows, the flow velocities are limited, since the model immanent Mach number needs to be kept sufficiently small. Therefore, a lowering kinematic viscosity, for achieving high Reynolds numbers for a given geometry, pushes the collision frequency towards the above-mentioned stability limit. It is possible to increase the value of $\omega$ by decreasing the size of lattices, however, it needs more computer resources [8].

Alternatively, using LBM-MRT increases stability limit and resolve the mentioned issue [9]-[17].

In the literature, there are comparative studies of the LBM-SRT and the LBM-MRT for lid driven cavity flows [2]-[18]. Those studies find that, the LBM-MRT is superior to the LBM-SRT at higher Reynolds number flow simulations, especially for numerical stability. Also, the LBM-SRT and the LBM-MRT produces accurate results for all Reynolds numbers. In addition that, the code using the LBM-MRT takes only $15 \%$ more CPU time than using the LBM-SRT.

In the previous work [2], [18], [19], LBM-SRT and LBM-MRT were compared, basically, for the accuracy issues. The stability properties were not explicitly addressed, besides a qualitative statement that LBM-MRT is more stable than the LBM-SRT. The originality of the present investigation compared to the previous work [2], [18], [19] lies especially therein that the stability properties of LBM-SRT and LBM-MRT are systematically and quantitatively compared over a large range of Reynolds and Mach numbers. Furthermore, for a better overall assessment of the accuracy, stability and convergence issues, the results are always compared with those of the well-established CFD code ANSYS-Fluent [20]. In the LBM-SRT, the collision frequency, and in the LBM-MRT, the $7^{\text {th }}$ and $8^{\text {th }}$ relaxation rates $\left(s_{7}=s_{8}\right)$ are related to the molecular kinematic viscosity. Therefore, collision frequency and $7^{\text {th }}$ relaxation rate are compared with changing Reynolds and Mach number as a stability limits of the LBM-SRT and the LBM-MRT respectively. Other relaxation times $\left(s_{0}, s_{1}, \ldots, s_{6}\right)$ for the 
LBM-MRT are taken from Razzaghian et al.,'s study [18], their work is taken as a reference in investigation of LBM-MRT stability limits.

\section{NUMERICAL METHODS}

\section{A. LBM with Single Relaxation Times (LBM-SRT)}

The lattice Boltzmann method is only applicable to the low Mach number hydrodynamics, because a small velocity expansion is used in derivation of the Navier-Stokes equation from lattice Boltzmann equation. It should be noted that the small Mach number limit is equivalent to incompressible limit [21].

The LBM method solves the microscopic kinetic equation for the particle distribution $f(x, v, t)$, where $x$ and $v$ is the particle position and the velocity vector respectively, in phase space $(x, v)$ and time $(t)$, where the macroscopic quantities which are velocity and density are obtained through moment integration of $f(x, v, t)$.

The discrete LBM-SRT equation, which is usually solved in two consecutive steps, i.e. in a "collision" and a following "streaming" step as provided below.

Collision step:

$$
\tilde{f}_{\alpha}(\vec{x}, t+\Delta t)=f_{\alpha}(\vec{x}, t)-\omega\left[f_{\alpha}(\vec{x}, t)-f_{\alpha}^{e q}(\vec{x}, t)\right]
$$

Streaming step:

$$
\tilde{f}_{\alpha}\left(\vec{x}+\bar{e}_{\alpha} \Delta t, t+\Delta t\right)=\tilde{f}_{\alpha}(\vec{x}, t+\Delta t)
$$

Note that, in the above " " denotes the post-collision values. It is obvious that collision process is completely localized, and the streaming step requires little computational effort by advancing the data from neighboring lattice points.

In (Eq. 1), $f_{\alpha}(\vec{x}, t)$ and $f_{\alpha}^{e q}(\vec{x}, t)$ are the particle distribution function and equilibrium particle distribution function of the $\alpha$-th discrete particle velocity $v, \vec{e}$ is a discrete velocity vector, and $\omega=\Delta t / \tau$ is the collision frequency. Note that $\tau$ is the collision relaxation time.

The 2-dimensional and 9-velocity (D2Q9) lattice model (Fig. 1) is used in the current study for simulating the steady lid driven cavity flow. The proposed D2Q9 lattice model obeys also incompressible limit. For isothermal and incompressible flows, the equilibrium distribution function can be derived as the following form [21].

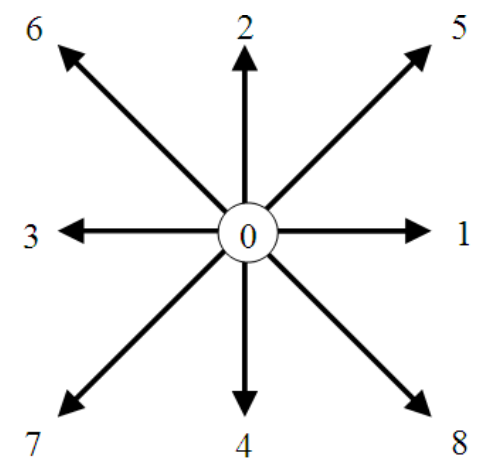

Fig. 1. D2Q9 lattice model.

$$
f_{\alpha}^{e q}(\vec{x}, t)=\rho w_{\alpha}\left[1+\frac{3}{c^{2}} \vec{e}_{\alpha} \cdot \vec{u}+\frac{9}{2 c^{4}}\left(\vec{e}_{\alpha} \cdot \vec{u}\right)^{2}-\frac{3}{2 c^{2}} \vec{u} \cdot \vec{u}\right]
$$

where $w_{\alpha}$ is a weighting factor, $\rho$ is the density, $\vec{u}$ is the fluid velocity and $c=\Delta x / \Delta t$, for square lattice, is the lattice speed, and $\Delta x(\Delta x=\Delta y), \Delta t$ are the lattice length and time step size. In addition, the discrete velocities for D2Q9 lattice model are

$$
\vec{e}_{\alpha}=c\left[\begin{array}{ccccccccc}
0 & 1 & 0 & -1 & 0 & 1 & -1 & -1 & 1 \\
0 & 0 & 1 & 0 & -1 & 1 & 1 & -1 & -1
\end{array}\right]
$$

and the values of weighting vectors $w_{\alpha}$ are

$$
w_{\alpha}=\left\{\begin{array}{ccc}
4 / 9 & \text { for } & \alpha=0 \\
1 / 9 & \text { for } & \alpha=1,2,3,4 \\
1 / 36 & \text { for } & \alpha=5,6,7,8
\end{array}\right.
$$

The macroscopic values are obtained from the following equations.

$$
\begin{gathered}
\rho=\sum_{\alpha=0}^{8} f_{\alpha}=\sum_{\alpha=0}^{8} f_{\alpha}^{e q} \\
\vec{u}=\frac{1}{\rho} \sum_{\alpha=0}^{8} \vec{e}_{\alpha} f_{\alpha}=\frac{1}{\rho} \sum_{\alpha=0}^{8} \vec{e}_{\alpha} f_{\alpha}^{e q} \\
P=\rho c_{s}^{2}
\end{gathered}
$$

where $P$ is the pressure and $c_{s}=c / \sqrt{3}$ is the lattice speed of sound. The viscosity of the simulated fluids is defined by

$$
v=\Delta t\left(\frac{1}{\omega}-\frac{1}{2}\right) c_{s}^{2}
$$

With the same way, collision frequency can be defined as $\omega=1 /\left(v /\left(c_{s}^{2} \Delta t\right)+1 / 2\right)$. The time step size $\Delta t$ is chosen in such a way to result in a lattice speed $c$ is unity, resulting in a lattice speed sound of $c_{s}=1 / \sqrt{3}$.

\section{B. LBM with Multiple Relaxation Times (LBM-MRT)}

As mentioned before, LBM with Multi Time Relaxation can improve the numerical stability of the LBM. The LBM-MRT collision model of $Q$ velocities on a $D$-dimensional lattice is written as [9]-[16].

$\mathbf{f}(\vec{x}+\vec{e} \Delta t, t+\Delta t)-\mathbf{f}(\vec{x}, t)=-\mathrm{M}^{-1} \cdot \hat{\mathrm{S}} \cdot\left[\mathbf{m}(\vec{x}, t)-\mathbf{m}^{e q}(\vec{x}, t)\right]$

where $\mathrm{M}$ is a $Q \times Q$ matrix which linearly transforms the distributions functions $\mathbf{f}$ to the velocity moments $\mathbf{m}$.

$$
\mathbf{m}=\mathbf{M} \cdot \mathbf{f} \quad \text { and } \quad \mathbf{f}=\mathbf{M}^{-1} \cdot \mathbf{m}
$$

the total number of discrete velocities $Q=1+b$ or $b$ for model with or without particle of zero velocity, respectively. 
$\hat{\mathrm{S}}$ is a non-negative $Q \times Q$ diagonal relaxation matrix, and the bold-face symbols denote the column vectors

$$
\begin{gathered}
\mathbf{f}(\vec{x}+\vec{e} \Delta t, t+\Delta t) \\
=\left(f_{0}(\vec{x}+\vec{e} \Delta t, t+\Delta t), \ldots, f_{b}(\vec{x}+\vec{e} \Delta t, t+\Delta t)\right)^{\mathrm{T}} \\
\mathbf{f}(\vec{x}, t)=\left(f_{0}(\vec{x}, t), \ldots, f_{b}(\vec{x}, t)\right)^{\mathrm{T}} \\
\mathbf{m}(\vec{x}, t)=\left(m_{0}(\vec{x}, t), \ldots, m_{b}(\vec{x}, t)\right)^{\mathrm{T}} \\
\mathbf{m}^{e q}(\vec{x}, t)=\left(m_{0}^{e q}(\vec{x}, t), \ldots, m_{b}^{e q}(\vec{x}, t)\right)^{\mathrm{T}}
\end{gathered}
$$

where $\mathrm{T}$ is transpose operator.

For D2Q9 lattice model, moments are listed below,

$$
\mathbf{m}=\left(\rho, e, \varepsilon, j_{x}, q_{x}, j_{y}, q_{y}, p_{x x}, p_{y y}\right)^{\mathrm{T}}
$$

where $\rho$ is the density, and $j_{x}=\rho u_{x}$ and $j_{y}=\rho u_{y}$ are $x$ and $y$ components of the flow momentum, respectively, which are the conserved moments in the system. Other moments are non-conserved moments and their equilibria are functions of the conserved moments in the system [9]-[16]. With this particular order of moments given above, the corresponding diagonal relaxation matrix of relaxation rates.

$$
\begin{aligned}
\hat{\mathrm{S}} & =\operatorname{diag}\left(s_{0}, s_{1}, s_{2}, s_{3}, s_{4}, s_{5}, s_{6}, s_{7}, s_{8}\right) \\
& =\operatorname{diag}\left(s_{\rho}, s_{e}, s_{\varepsilon}, s_{j_{x}}, s_{q_{x}}, s_{j_{y}}, s_{q_{y}}, s_{p_{x x}}, s_{p_{x y}}\right)
\end{aligned}
$$

The equilibria of the non-conserved moments

$$
\begin{gathered}
m_{1}^{e q}=e^{e q}=-2 \rho+3\left(j_{x}^{2}+j_{y}^{2}\right), \\
m_{2}^{e q}=\varepsilon^{e q}=\rho-3\left(j_{x}^{2}+j_{y}^{2}\right) \\
m_{4}^{e q}=q_{x}^{e q}=-j_{x}, m_{6}^{e q}=q_{x}^{e q}=-j_{y}, \\
m_{7}^{e q}=p_{x x}^{e q}=\left(j_{x}^{2}-j_{y}^{2}\right), m_{8}^{e q}=p_{x y}^{e q}=j_{x} j_{y}
\end{gathered}
$$

with the orderings of the discrete velocities and corresponding moments given above for D2Q9 lattice model, the transform matrix $\mathrm{M}$ in (10) is

$$
M=\left(\begin{array}{ccccccccc}
1 & 1 & 1 & 1 & 1 & 1 & 1 & 1 & 1 \\
-4 & -1 & -1 & -1 & -1 & 2 & 2 & 2 & 2 \\
4 & -2 & -2 & -2 & -2 & 1 & 1 & 1 & 1 \\
0 & 1 & 0 & -1 & 0 & 1 & -1 & -1 & 1 \\
0 & -2 & 0 & 2 & 0 & 1 & -1 & -1 & 1 \\
0 & 0 & 1 & 0 & -1 & 1 & 1 & -1 & -1 \\
0 & 0 & -2 & 0 & 2 & 1 & 1 & -1 & -1 \\
0 & 1 & -1 & 1 & -1 & 0 & 0 & 0 & 0 \\
0 & 0 & 0 & 0 & 0 & 1 & -1 & 1 & -1
\end{array}\right)
$$

For D2Q9 lattice model, the shear viscosity $v$, which is same in (Eq. 9), but in this formula, $7^{\text {th }}$ and $8^{\text {th }}$ relaxation rates used instead of collision frequency and the bulk viscosity $\zeta$ are;

$$
v=\Delta t\left(\frac{1}{s_{7}}-\frac{1}{2}\right) c_{s}^{2}, \zeta=\frac{\Delta t}{2}\left(\frac{1}{s_{1}}-\frac{1}{2}\right) c_{s}^{2}
$$

For D2Q9 lattice model, it is required that $s_{7}=s_{8}$ and $s_{4}=s_{6}$. Obviously the relaxation rates $s_{0}, s_{3}$ and $s_{5}$ for the conserved moments $\left(\rho, j_{x}, j_{y}\right)$ have no effect for the model.. The other relaxation rates, $s_{2}$ (for $\varepsilon$ ) and $s_{4}=s_{6}$ (for $q_{x}$ and $q_{y}$ ) do not affect the hydrodynamics in the lowest order approximation and only affect the small scale behavior of the model. Also, the relaxation rates $\left(s_{4}=s_{6}\right)$ can affect the accuracy boundary conditions [22], [23].

The LBM-MRT model can reproduce the same viscosity with the LBM-SRT model, if we set $s_{7}=s_{8}=\omega$. And, the rest of the relaxation parameters $\left(s_{1}, s_{2}, s_{4}\right.$ and $\left.s_{6}\right)$ can be chosen more flexibly [11].

As we mentioned before, in the LBM-MRT calculations, the study of Razzaghian et al. [18] is taken as a reference study, Thus, we will use $s_{0}=s_{3}=s_{5}=1, s_{1}=s_{2}=1.4$, $s_{4}=s_{6}=1.2$ and $s_{7}=s_{8}=\omega$ for LBM-MRT calculations . And, we will compare the stability limits of the LBM-SRT and LBM-MRT using collision frequency $(\omega)$ and $7^{\text {th }}$ relaxation rate $\left(s_{7}=s_{8}\right)$ for lid driven cavity flow, respectively.

\section{RESULTS AND DISCUSSION}

\section{A. Lid Driven Cavity Flow}

The lid driven cavity flows are investigated. The geometry and boundary conditions of the lid driven cavity flow are sketched in Fig. 2. Where $u_{x}$ and $u_{y}$ are $x$ and $y$ component of the flow velocity, and $u_{0}$ is the boundary value.

Computations are performed for Reynolds numbers (Re), which are based on inlet velocity $\left(u_{0}\right)$ and hydraulic diameter $(H)$ varying within the range 200 and 2000. Mach numbers $(\mathrm{Ma})$ which are based on inlet velocity $\left(u_{0}\right)$ and speed of sound $\left(c_{s}\right)$ are varied between 0.09 and 0.54 . In order to acquire Mach numbers, inlet velocities are varied between 0.0519 and 0.3117 . For each computation, various values of the collision frequency $(\omega)$ for LBM-SRT and $7^{\text {th }}$ and $8^{\text {th }}$ relaxation rates $\left(s_{7}=s_{8}\right)$ for LBM-MRT are used, for detecting the highest possible value for a stable solution.

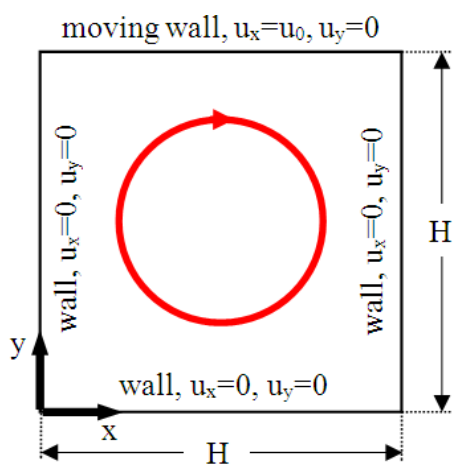

Fig. 2. Lid driven cavity flow. 


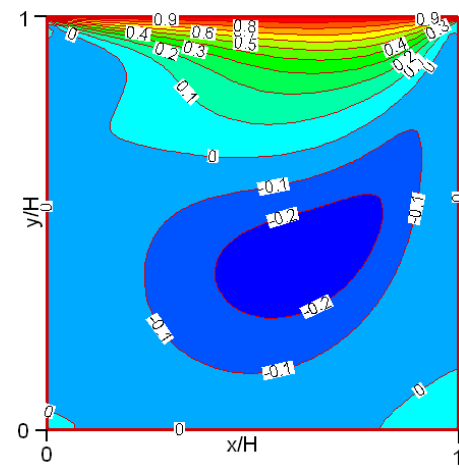

(a)

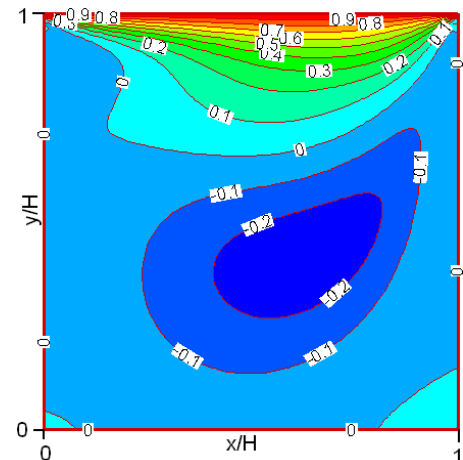

(b)

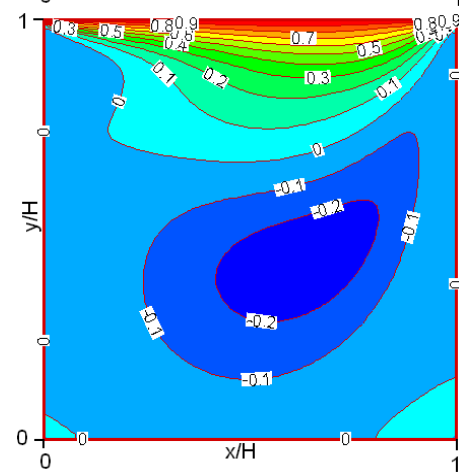

(c)

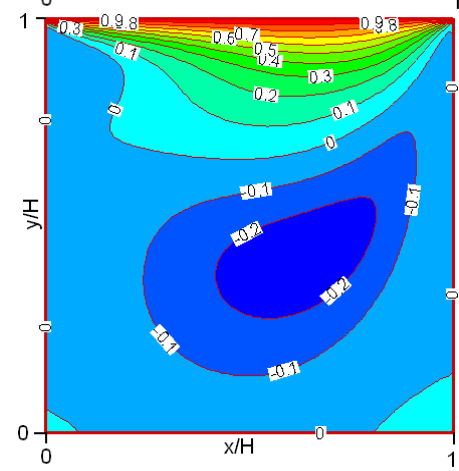

(d)

Fig. 3. LBM predicted non dimensional velocity $\left(u_{x} / u_{0}\right)$ contours for $\mathrm{Re}=200$ and lid driven cavity flow: (a) LBM-SRT, Ma=0.09, (b) LBM-MRT, $\mathrm{Ma}=0.09$, (c) LBM-SRT, Ma=0.54, (d) LBM-MRT, Ma=0.54.

Incompressible LBM formulations are used both LBM-SRT and LBM-MRT. Ansys-Fluent discretizes the incompressible Navier-Stokes equations with Finite Volume Method. Therefore, for validation LBM results, well-established CFD code, Ansys-Fluent is used. The same mesh sizes are used, of course, for validation

Fig. 3 displays the predicted contours of nondimensional $u_{x}$ velocity component for $\mathrm{Re}=200$ and lid driven cavity flow, predicted for different Mach numbers, and for the LBM-SRT and the LBM-MRT. Comparing the solution for $\mathrm{Re}=200$, one can see that Mach number variations within the considered range does not remarkably affect from the flow field for the LBM-SRT and the LBM-MRT. As also can be seen from the figure, LBM-SRT and LBM-MRT predictions are very close each other.

Fig. 4 displays the predicted contours of nondimensional $u_{x}$ velocity component for $\mathrm{Re}=2000$ and lid driven cavity flow, predicted for different Mach numbers, and for the LBM-SRT and LBM-MRT. The main recirculation structure gets more symmetric for $\mathrm{Re}=2000$, as expected based on previous studies on this typical benchmark flow problem Comparing the solution for $\mathrm{Re}=2000$, one can see that Mach number variations within the considered range does not remarkably affect from the flow field for LBM-SRT and LBM-MRT. Additionally, there is unnoticeable difference between predictions of LBM-SRT and LBM-MRT

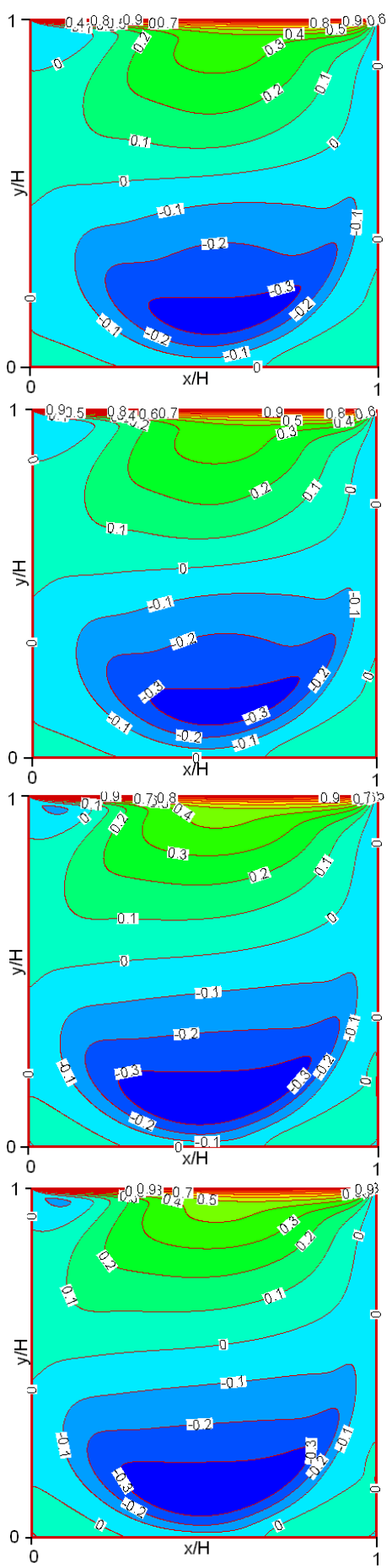

(a)

(b)

(c)

(d)

Fig. 4. LBM predicted non dimensional velocity $\left(u_{x} / u_{0}\right)$ contours for

$\mathrm{Re}=2000$ and lid driven cavity flow: (a) LBM-SRT, Ma=0.09, (b) LBM-MRT, Ma=0.09, (c) LBM-SRT, Ma=0.54, (d) LBM-MRT, Ma=0.54. 

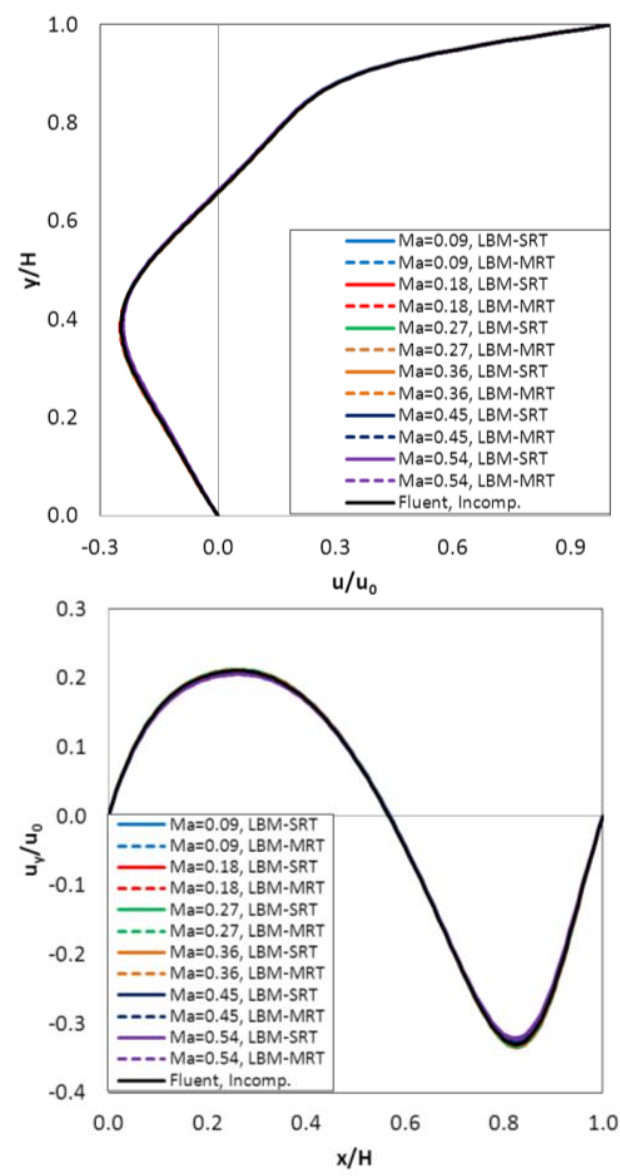

(b)

Fig. 5. Non dimensional velocity profiles for $\mathrm{Re}=200$, (a) $u_{x}$ velocity at $x=H / 2$, (b) $u_{y}$ velocity at $y=H / 2$.

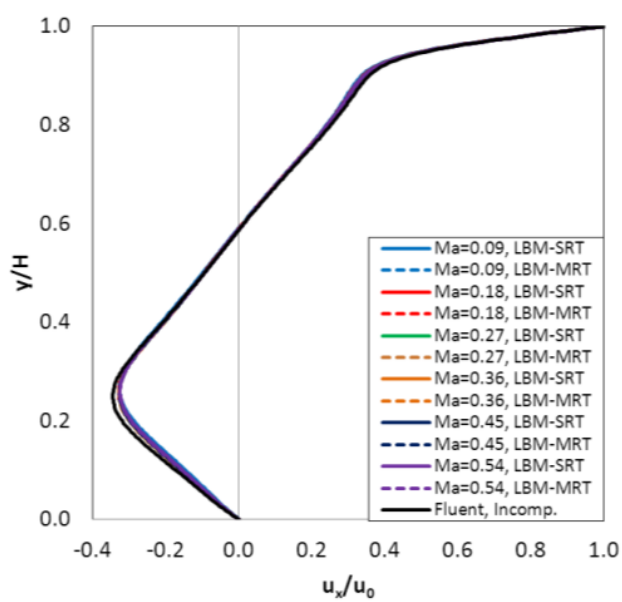

(a)

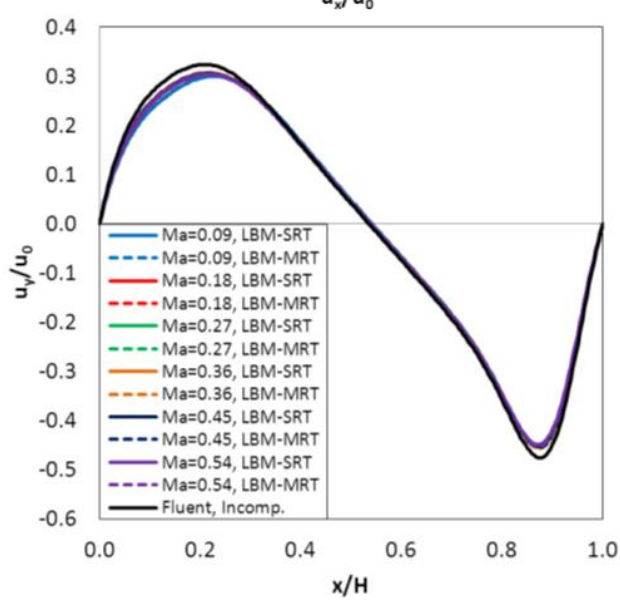

(b)

Fig. 6. Non dimensional velocity profiles for $\mathrm{Re}=500$, (a) $u_{x}$ velocity at $x=H / 2$, (b) $u_{y}$ velocity at $y=H / 2$.

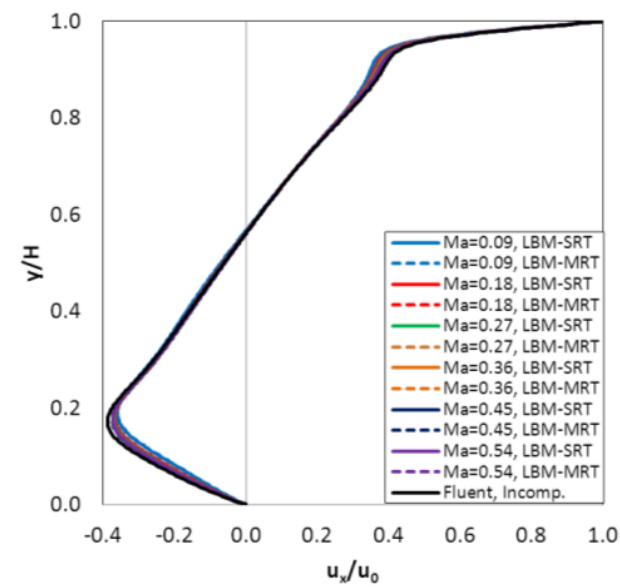

(a)

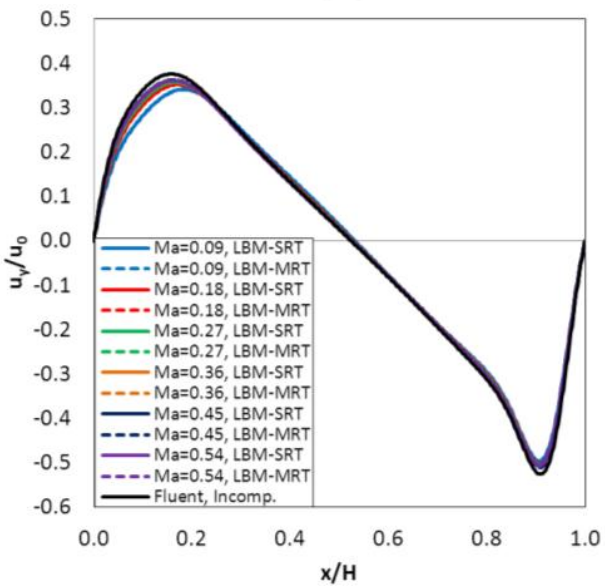

(b)

Fig. 7. Non dimensional velocity profiles for $\mathrm{Re}=1000$

(a) $u_{x}$ velocity at $\mathrm{x}=\mathrm{H} / 2$, (b) $u_{y}$ velocity at $y=H / 2$.
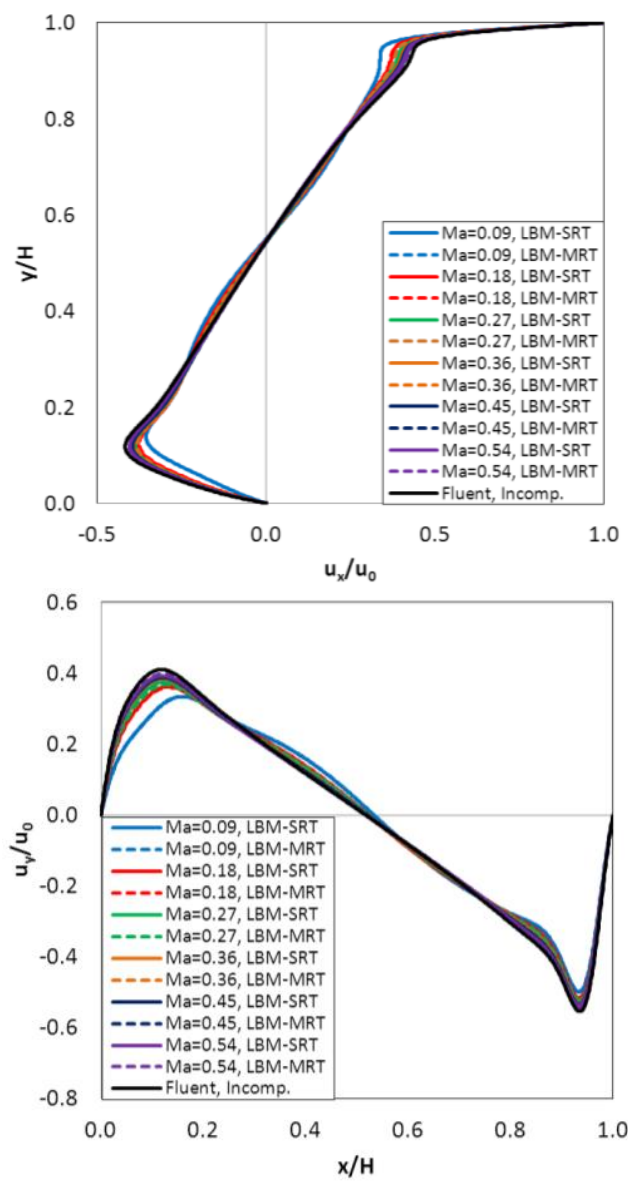

Fig. 8. Non dimensional velocity profiles for $\mathrm{Re}=2000$, (a) $u_{x}$ velocity at $x=H / 2$, (b) $u_{y}$ velocity at $y=H / 2$. 
Fig. 5(a) compares the predicted $u_{x}$ velocity profiles along a vertical line at $x / H=1 / 2$ for $\operatorname{Re}=200$. The $u_{y}$ velocity profiles for the same Reynolds number, along a horizontal line at $y / H=1 / 2$ are compared in Fig. 5(b). In Fluent computations, $2^{\text {nd }}$ Order Upwind scheme have been used as discretization scheme. Fluent and all LBM predictions are displayed in the figures. One can see that the all LBM predictions (from $\mathrm{Ma}=0.09$ and $\mathrm{Ma}=0.54$ ) are quite close each other and agree very well with the Fluent predictions for $\mathrm{Re}=200$.

Fig. 6(a), Fig. 7(a) and Fig. 8(a) present the predicted $u_{x}$ velocity profiles along a vertical line at $x / H=1 / 2$ for $\mathrm{Re}=500$, 1000 and 2000 respectively. The predicted $u_{y}$ velocity profiles along a horizontal line at $y / H=1 / 2$ are compared in Fig. 6(b), Fig. 7(b) and Fig. 8(b) for $\mathrm{Re}=500,1000$ and 2000 respectively. One can see from the figures that as the Reynolds number increases, the difference between the LBM and Fluent predictions becomes larger. The largest differences between the LBM and the Fluent computations are observed at $\mathrm{Ma}=0.09$ for all Reynolds number. As it can also be seen from the figures, for all Reynolds numbers and all

Mach numbers, the predictions of the LBM-SRT and the LBM-MRT are quite close each other.

Based on the lid driven cavity flow, converge behaviors of the present LBM-SRT and LBM-MRT code and Fluent are also compared in Fig. 9, for $\mathrm{Re}=1000$ and $\mathrm{Ma}=0.27$. For a better comparability, the same criteria, namely the percentage variation (which indicated as $\% \varepsilon$ in the figures) of a variable at a given monitor point is taken as the indicator of the convergence, for all codes. For general variable $\varphi$ (which can be $u_{x}$ or $\left.u_{y}\right)$, this is computed from

$$
\% \varepsilon=100 \times \frac{\varphi^{n+1}-\varphi^{n}}{\varphi^{n}}
$$

In (18), the parameter $n$ denotes the iteration number. Obviously, the same grids are used, and computations are started from the same initial velocity field distributions (zero velocity everywhere in the flow field). Of course, the same computer is used for all computations. For the Fluent computations, the Simple pressure-correction procedure is used. For the under relaxation factors, the default values are applied for all variables [20]. As can be seen in Fig. 9, both Lattice Boltzmann computations (the LBM-SRT and the LBM-MRT) show, in general, a better overall convergence rate (according to present definition described by (Eq.18)). On the other hand, all the Lattice Boltzmann Method results exhibit some "wiggles" along the way of convergence. The residuals obtained by the Ansys-Fluent code exhibit a more smooth behavior. As it can also be seen in Fig. 9, converge of the LBM-SRT is achieved approximately $\% 10$ earlier than the LBM-MRT.

\section{B. Stability Limits}

For a range of Reynolds ( $200 \leq \operatorname{Re} \leq 2000$ ), and Mach $(0.09 \leq \mathrm{Ma} \leq 0.54)$ numbers, different values of collision frequency $(\omega)$ for the LBM-SRT and $7^{\text {th }}$ and $8^{\text {th }}$ relaxation rates $\left(s_{7}=s_{8}\right.$ ) for the LBM-MRT are applied, for detecting the maximum allowed value beyond which the solution becomes unstable, i.e, no converged steady-state solution can be obtained. Theoretically, it is obvious that the collision frequency or $7^{\text {th }}$ (or $8^{\text {th }}$ ) relaxation rates are not allowed to take the value 2 , but needs to be smaller.

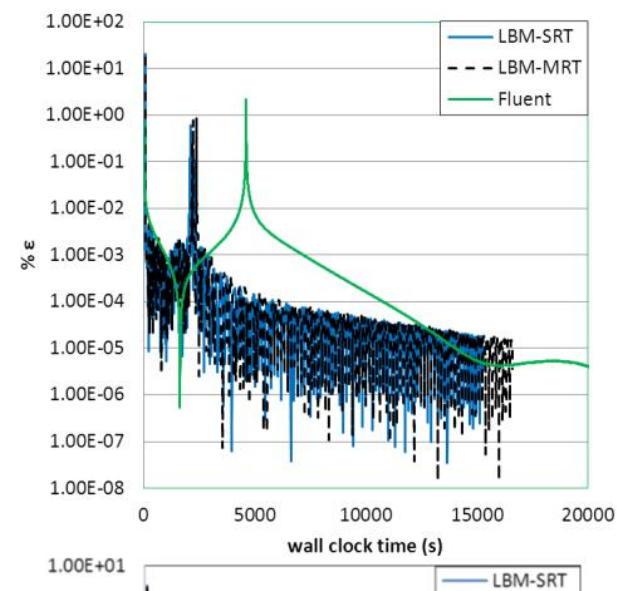

(a)

(b)

Fig. 9. Converge behavior ( $\mathrm{Re}=1000$ and $\mathrm{Ma}=0.27)$ : (a) $\% \varepsilon$ in $u_{x}$ at $x=H / 2$, $y=3 H / 4$, (b) $\% \varepsilon$ in $u_{x}$ at $x=H / 2, y=H / 4$, (c) $\% \varepsilon$ in $u_{y}$ at $x=H / 4, y=H / 2$, (d) $\% \varepsilon$ in $u_{y}$ at $x=3 H / 4, y=H / 2$. 
For the LBM-SRT, the predicted maximum allowed collision frequency for a stable solution (the solid lines) are presented in Fig. 10, as a function of Mach number, for different values of the Reynolds number. As can be seen from Figure 10, the maximum allowed collision frequency $(\omega)$ values decrease with Reynolds number, whereas for a given Reynolds number, also a decrease with the Mach number is predicted.

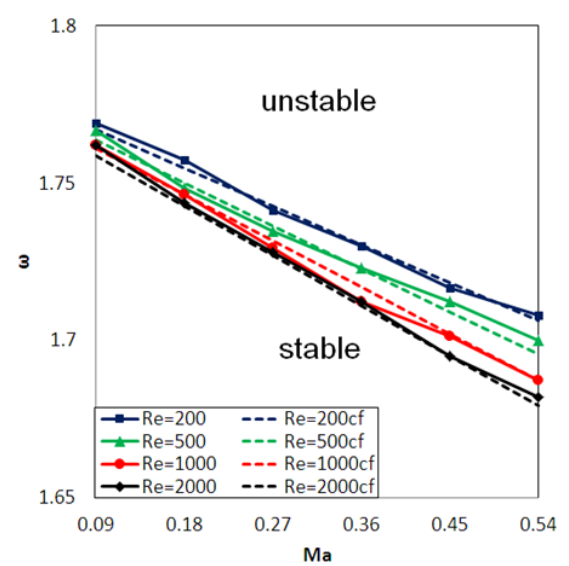

Fig. 10. Predicted maximum $\omega$ values (LBM-MRT) for stable solution:

The dashed lines and suffix "cf" refer to the curve of (19) and Table I.

For the LBM-MRT, the predicted maximum $7^{\text {th }}$ and $8^{\text {th }}$ relaxation rates for a stable solution (the solid lines) are presented in Fig. 11, as a function of Mach number, for different values of Reynolds number. The maximum $7^{\text {th }}$ and $8^{\text {th }}$ relaxation rates increase with increasing Reynolds number and with decreasing Mach number.

The curves mostly exhibit a linear like variation with the Mach number for the LBM-SRT calculations. Thus, a trial has been given to fit a linear curve to the predicted data, the coefficients being functions of the Reynolds number, which can be expressed as;

$$
\omega_{\text {MAX }}=a_{1}(\operatorname{Re}) \times M a+a_{2}(\operatorname{Re})
$$

The coefficients $a_{1}(\mathrm{Re})$ and $a_{2}(\mathrm{Re})$ of (19), which are obtained by curve fitting to the predicted data are presented in Table I. The linear curves predict by (19) are also displayed in Fig. 10, as the dashed lines, where corresponding legends are designated by the suffix "cf" (for "curve fitting") after the corresponding Re value.

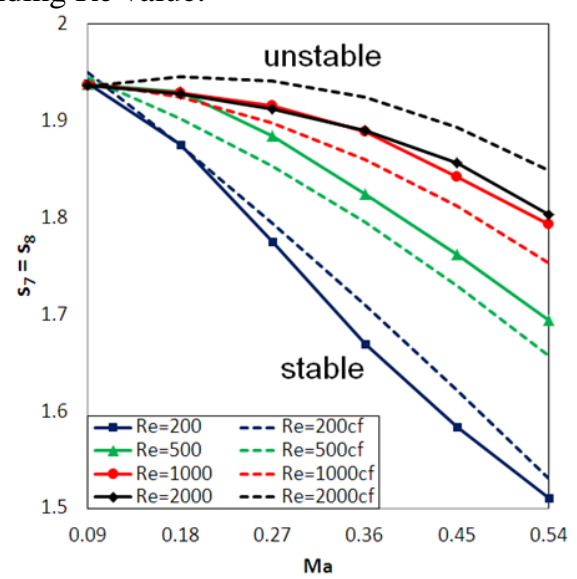

Fig. 11. Predicted maximum $s_{7}\left(s_{7}=s_{8}\right)$ values (LBM-MRT) for stable solution: The dashed lines and suffix "cf" refer to the curve of (20) and Table 2.

\begin{tabular}{|c|c|}
\hline & LBM-SRT \\
\hline$a_{1}$ & $-0.018 \ln (\mathrm{Re})-0.396$ \\
\hline$a_{2}$ & $-0.002 \ln (\operatorname{Re})+1.7897$ \\
\hline
\end{tabular}

For the LBM-MRT calculations, the curves mostly exhibit a $2^{\text {nd }}$ order polynomial like variation with the Mach number. Therefore, a trial has been presented to fit a $2^{\text {nd }}$ order polynomial curve to the predicted data, the coefficients being functions of the Reynolds number, which can be expressed as;

$$
s_{7, M A X}=s_{8, M A X}=b_{1}(\operatorname{Re}) \times M a^{2}+b_{2}(\operatorname{Re}) \times M a+b_{3}
$$

The coefficients $b_{1}(\mathrm{Re}), b_{2}(\mathrm{Re})$ and $b_{3}(\mathrm{Re})$ of $(20)$, which are obtained by curve fitting to the predicted data are presented in Table 2 . The $2^{\text {nd }}$ order polynomial curves predict by (20) are also displayed in Fig. 11, as the dashed lines, where corresponding legends are designated by the suffix "cf" after the corresponding Re value,

\begin{tabular}{cc} 
TABLE II: Coefficients $b_{1}(\mathrm{Re}), b_{2}(\mathrm{Re})$ and $b_{3}(\mathrm{Re})$ of $(20)$ \\
\hline \hline$b_{1}$ & $-0.26 \ln (\mathrm{Re})+1.1415$ \\
$b_{2}$ & $0.04856 \ln (\mathrm{Re})-3.3577$ \\
$b_{3}$ & $-0.048 \ln (\mathrm{Re})+2.2777$ \\
\hline \hline
\end{tabular}

\section{CONCLUSION}

Incompressible steady state formulations of the LBM-SRT and the LBM-MRT are applied to laminar flows for Reynolds numbers between 200 and 2000, where the Mach number is also varied between 0.09 and 0.54 . The lid driven cavity flow problem is analyzed. Stability limits, in terms of the maximum allowed collision frequency ( $\omega$ ) for LBM-SRT and $7^{\text {th }}$ and $8^{\text {th }}$ relaxation rates $\left(s_{7}=s_{8}\right)$, as a function of Reynolds and Mach numbers are explored. It is observed that, for low Mach and high Reynolds number the LBM-MRT is more stable than LBM-SRT. Collision frequency decreases with increasing Reynolds and Mach numbers for the LBM-SRT. However, $7^{\text {th }}$ and $8^{\text {th }}$ relaxation rates decrease with decreasing Reynolds numbers and with increasing Mach numbers. Comparisons with the general purpose, finite-volume based CFD code, using incompressible formulation has served as a validation of the present Lattice Boltzmann Method (both the LBM-SRT and the LBM-MRT) based code, at the same time confirming that the present incompressible Lattice Boltzmann formulation predicts flow field that behave sufficiently incompressible for the considered range of Mach numbers. Also, converge behavior of the both Lattice Boltzmann codes (both LBM-SRT and LBM-MRT) and finite volume based CFD code are explored. It is observed that, all Lattice Boltzmann codes are much faster than finite volume based CFD code. Also, convergence speed of the LBM-SRT is better (approximately \%10) than LBM-MRT with using same grid size. 


\section{REFERENCES}

[1] F.J. Higuera and J. Himenez, "Boltzmann Approach to Lattice Gas Simulation," Europhysics Letter, vol. 9, pp. 663-668, 1989.

[2] J. S. Wu and Y. L. Shao, "Assessment of SRT and MRT scheme in parallel lattice boltzmann method for lid-driven cavity flows," in Proc. the 10th National Computational Fluid Dynamics Conference, Hua-Lien, 2003.

[3] F. J. Higuera, S. Succi, and R. Benzi, "Lattice gas-dynamics with enchanced," Europhysics Letter, vol. 9, pp. 345-349, 1989.

[4] P. L. Bhatnagar, E. P. Groos, and M. Krook, "A model for collision process in gases I. small amplitude process in charged and neutral one component system,” Physical Review, vol. 94, pp. 511-525, 1954.

[5] Y. Qian, D. D'Humières, and P. Lallemand, "Lattice BGK models for navier-stokes Equation," Europhysics Letter, vol. 17, pp. 479-484, 1992.

[6] S. Succi, The Lattice Boltzmann Equation for Fluid Dynamics and Beyonds, Oxford, Calderon Press, 2001.

[7] M. Fink, "Simulation von Nasenströmungen mit Lattice-BGK-Methoden," Ph.D. dissertation, Essen-Duisburg University, Germany, 2007.

[8] A. C. Benim, E. Aslan, and I. Taymaz, "Investigation into LBM analyses of incompressible laminar flows at high reynolds numbers," WSEAS Transactions on Fluid Mechanics, vol. 4, no. 4, pp. 107-116, 2009.

[9] D. d'Humières, "Generalized lattice boltzmann equation," AIAA Rarefied Gas Dynamics: Theory and simulations, vol. 159, pp. 450-458, 1992.

[10] D. d'Humières, I. Ginzburg, M. Krafczyk, P. Lallemand, and L. S. Luo, "Multiple relaxation time lattice boltzmann models in three dimensions," Philosophical Transactions The Royal Society London, vol. 360, pp. 437-451, 2002.

[11] P. Lallemand and L. S. Luo, "Theory of the lattice boltzmann method: dispersion isotropy galilean invariance and stability," Physical Review $E$, vol. 61, no. 1, pp. 775-719, 2000.

[12] P. Lallemand and L. S. Luo, "Theory of the lattice boltzmann method: acoustic and thermal properties in in two and three dimensions," Physical Review E, vol. 68, pp. 036706-1-036706-25, 2003.

[13] A. A. Mohamad, Lattice Boltzmann Method, Fundementals, Applications with Computer Codes, London, Springer, 2011.

[14] M. A. Moussaoui, A. Mezrhab, H. Naji, and M. E. Gabaoui, "Prediction of heat transfer in a plane channel built-in three heated square obstacles using an mrt lattice boltzmann method," in Proc. the Sixth International Conference on Computational Heat and Transfer, Guanzhou, China, 2009, pp.176-181.

[15] M. A. Moussaoui, M. Jami, A. Mezrhab, and H. Naji, "MRT-lattice boltzmann simulation of forced convection in a plane channel with an inclined square cylinder," International Journal of Thermal Sciences, vol. 49, pp. 131-142, 2010.

[16] H. Yu, L. S. Luo, and S. S. Girimaji, "LES of turbulent square jet flow using an MRT lattice boltzmann model," Computers \& Fluids, vol. 35, pp. 957-965, 2006.

[17] M. Pourtousi, M. Razzaghian, A. Saftari, and A. N. Darus, "Simulation of fluid flow inside a back-ward-facing step by MRT-LBM," in Proc.

2012 International Conference on Fluid Dynamics and Technologies, Singapore, 2012, pp. 130-135.

[18] M. Razzaghian, M. Pourtousi, and A. N. Darus, "Simulation of flow in lid driven cavity by MRT and SRT," in Proc. International Conference on Mechanical and Robotics Engineering, Phuket, 2012, pp. 94-97.

[19] J. S. Wu and Y. L. Shao, "Simulation of lid driven cavity flow by parallel lattice boltzmann method using multi relaxation time scheme," International Journal for Numerical Methods in Fluids, vol. 46, pp. 921-937, 2004.

[20] Ansys-Fluent 12.0 Users's Guide, Ansys Inc., 2009.

[21] X. He and L. S. Luo, "Lattice boltzmann model for incompressible navier-stokes equation” Journal of Statiscal Physics, vol. 88, no. 3-4, pp. 927-944, 1997

[22] D. A. Wolf-Gladrow, Lattice Gas Cellular Automata and Lattice Boltzmann Models, Berlin, Springer, 2005.

[23] Y. Peng and L. S. Luo, "A comparative study of Immersed-Boundary and Interpolated Bounce-back methods, Progress in Computational Fluid Dynamics, vol. 8, no. 1-4, pp. 156-157, 2008.

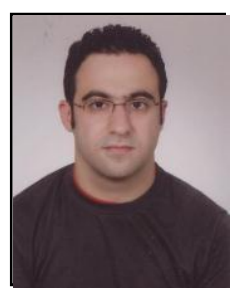

and LBM.
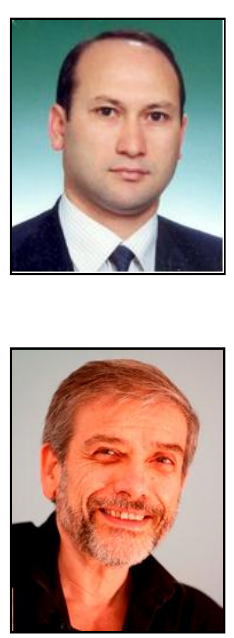

Sciences, Germany.
E. Aslan received his $\mathrm{BSc}$, MSc and $\mathrm{PhD}$ in mechanical engineering from the University of Sakarya, Turkey.

He is currently an assistant professor of Mechanical Engineering Department at University of Istanbul. He teaches undergraduate and graduate courses. His research area fluid mechanics, heat transfer, aerodynamics, numerical methods on fluid flow and heat transfer such as Finite Volume Method (FVM)

I. Taymaz received his BSc and MSc in mechanical engineering from the Technical University of Istanbul, and his PhD form University of Sakarya, Turkey.

He is currently an associate professor of Mechanical Engineering Department at University of Sakarya. He teaches undergraduate and graduate courses in automotive division. His research areas of interest include advanced technologies in engines for automotive industry.

C. Benim received his BSc and MSc in mechanical engineering from the Bosphorus University of Istanbul, Turkey and his $\mathrm{PhD}$ form University of Stuttgart, Germany, in 1988.

Following a post-doctoral period at the University of Stuttgart, he joined ABB Turbo Systems Ltd. in Baden, Switzerland, in 1990. He was the manager of the "Computational Flow and Combustion Modelling" group, since January 1996, he is a professor of energy technology at the Düsseldorf University of Applied 